\title{
Spatiotemporal changes of epidemics and their relationship with human living environments in China over the past 2200 years
}

\author{
Shengsheng GONG ${ }^{1}$, Haichao $\mathrm{XIE}^{2}$ \& Fahu $\mathrm{CHEN}^{2,3^{*}}$ \\ ${ }^{1}$ College of Urban and Environmental Sciences/Research Institute of Sustainable Development, Central China Normal University, Wuhan \\ 430079, China; \\ ${ }^{2}$ Key Laboratory of Alpine Ecology, Institute of Tibetan Plateau Research, Chinese Academy of Sciences, Beijing 100101, China; \\ ${ }^{3}$ CAS Center for Excellence in Tibetan Plateau Earth Sciences, Chinese Academy of Sciences, Beijing 100101, China
}

Received February 26, 2020; revised March 7, 2020; accepted March 31, 2020; published online June 2, 2020

Citation: Gong S, Xie H, Chen F. 2020. Spatiotemporal changes of epidemics and their relationship with human living environments in China over the past 2200 years. Science China Earth Sciences, 63: 1223-1226, https://doi.org/10.1007/s11430-020-9608-x

Epidemics are biological disasters resulting from the widespread occurrence of acute and severe infectious diseases that are normally caused by viruses, bacteria and parasites. They are amongst the most destructive of all natural disasters suffered by humans (Gong, 2019). The pathogens that cause epidemics have always existed in nature, understanding the spatiotemporal distribution of past epidemics is important for preventing and controlling future epidemics.

\section{A review of progress in the study of epidemics during the Chinese historical period}

The Chinese character "Yi", meaning "epidemic", was inscribed on oracle bones as long ago as the Yin-Shang period (1300B.C.E.-1046B.C.E.), and the earliest extant description of the relationship between epidemic diseases and animals in China is in the oldest physical geographical monograph of China, entitled Shan Hai Jing. Subsequently, historical documents such as official histories, chronicles, memoirs, archives, anthologies, medical records, newspapers, and periodicals, also provide valuable historical materials for the study of epidemics in China.

During the past 20 years, the historical medical geography research team of Central China Normal University in Wuhan

\footnotetext{
* Corresponding author (email: fhchen@itpcas.ac.cn)
}

has focused on the study of epidemic history in China (Gong, 2003, 2019), and it recently produced a book entitled Annals of Epidemics in China Over the Past 3000 Years (Gong, 2019). More than 8931 historical documents and 6622 references were used and it is the most comprehensive compilation of documentary evidence of epidemics in China. More details about this book please see Appendix 1 (https://link.springer. com).

Historical records of epidemics predating the Qin Dynasty are rare and therefore in the present study, based on the data of Gong (2019), we discuss the spatiotemporal pattern and possible mechanisms of epidemics since the Qin Dynasty (220B.C.E.-AD1949).

\section{Epidemics in China over the past 2200 years}

During the past 2200 years, the frequency of epidemics in China has gradually increased (Figure 1a). During the Han Dynasty (206B.C.E.-AD220), epidemics occurred roughly once every two decades; during the Wei, Jin, Southern and Northern dynasties (AD220-AD581), they occurred every $\sim 5$ years; during the Northern Song Dynasty (AD960AD1127), every $\sim 3$ years; and during the period of the Republic of China (R.O.C.), roughly every year. In terms of epidemics the entire historical period can be divided into three stages. In the first stage (220B.C.E.-AD1000), the 


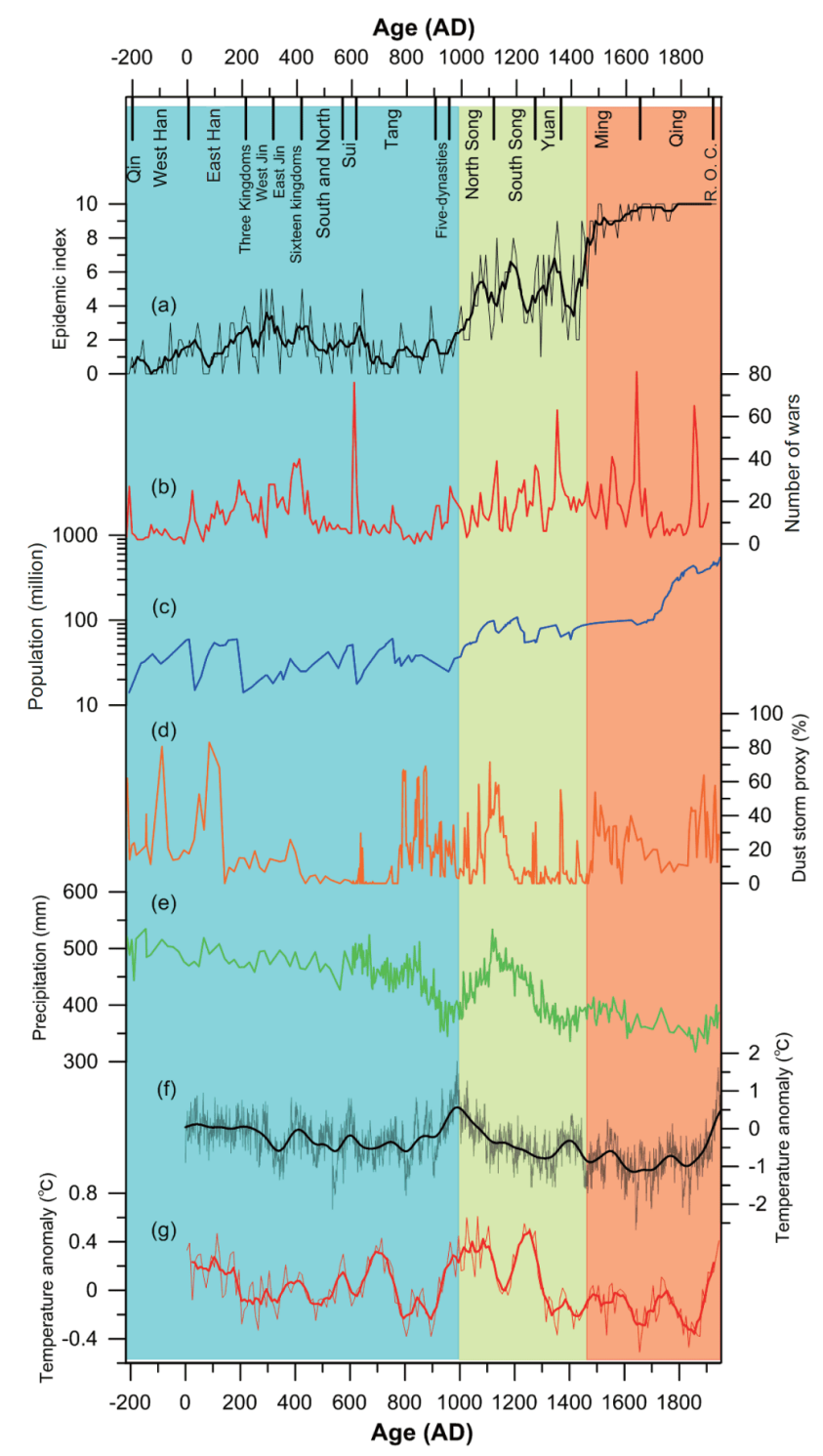

Figure 1 Changes in the frequency of epidemics in China over the past 2200 years. The epidemic index (a) modified from Gong (2019), which is the number of years per decade in which epidemics occur. (b) Number of wars per decade in China. (c) Population of China. (d) Dust storm proxy for North China. (e) Precipitation in the East Asian monsoon region. (c)-(e) are modified from Chen et al. (2020). Temperature anomaly records for the Northern Hemisphere (f) and China (g). (f)-(g) are modified from Ge et al. (2013). The principal dynasties of China are shown at the top of the figure. The thick line in Figure 1a is the 50-year moving average. The incidence of wars shown in Figure $1 \mathrm{~b}$ is calculated from the Tabulation of Wars in Ancient China (1985).

epidemic index (the epidemic index is the number of years in which epidemics occurred per decade) was less than 5, with an average of 1.46. During the Han and Tang dynasties, the epidemic index was the lowest, and during several decadal intervals there were no epidemics. During the late Eastern Han Dynasty and the Wei, Jin, and Northern and Southern dynasties, the epidemic index was relatively high, resulting in the first epidemic peak during the past 2200 years. During the second stage (AD1000-AD1450), epidemics occurred almost once every decade, and the epidemic index increased substantially, averaging 4.64. Only during the Southern Song Dynasty (AD1240-AD1280) and the early Ming Dynasty (AD1430-AD1440) were epidemics infrequent. During the third stage (AD1450-AD1949) epidemics were the most frequent during the past 2200 years, with an average epidemic index of 9.44, and within the territory of modern China, epidemics occurred almost every year (Figure 1a). During the first two stages there is a roughly centennial-scale cyclicity in the number of epidemics, each cycle characterized by a gradual increase followed by a rapid decrease, which is more clearly evident in the smoothed record (Figure 1a). In addition to the long-term increasing trend and high-frequency fluctuations, there are also temporal changes in the seasonality of epidemics. More details please see Appendix 2.

\section{Changes in the spatial distribution of epi- demics in China during the past 2200 years}

During the last 2200 years, there were pronounced spatial differences in the number of epidemic years per province in China, with the largest number in the southeast, followed by central China, and with the smallest number in the northwest (Figure 2a). During the past 300 years, since the spatial resolution of epidemics can be increased to county level, the spatial differences in epidemics can be evaluated on the basis of the modern county administrative divisions of China in 2011 (Figure 2b). Among all 2435 counties in China today, 2211 of them have experienced epidemics, which means that as many as $90.8 \%$ of the counties experienced epidemics during the past 300 years. During this interval there were a cumulative total of 27511 counties which experienced epidemics; the proportion for densely populated southeastern China is $88.03 \%$, and that for sparsely populated northwestern China is $11.97 \%$. Of the 1926 counties in southeastern China, 1860 of them have experienced epidemics, with a coverage of $96.6 \%$. In contrast, only 351 out of 509 counties in northwestern China experienced epidemics, with a coverage of $69.0 \%$. Thus the spatial distribution of epidemics was essentially the same as that of the human population. In contrast to other natural disasters, epidemics are population density dependent; therefore, coastal areas with a high population density and fast economic development (such as the Yangtze River Delta), the transport hub cities with high population mobility (such as Lanzhou, Wuhan, and Shanghai), and major traffic routes (major road routes such as the Hexi Corridor in Gansu, and waterways such as the Grand Canal) had a high risk of epidemics (Figure 2).

The frequency of epidemics in China has also changed spatially, with the epidemic center moving from north to south over the past 2200 years. During the Pre-Qin and Han dynasties, the major epidemic-affected areas were located in 


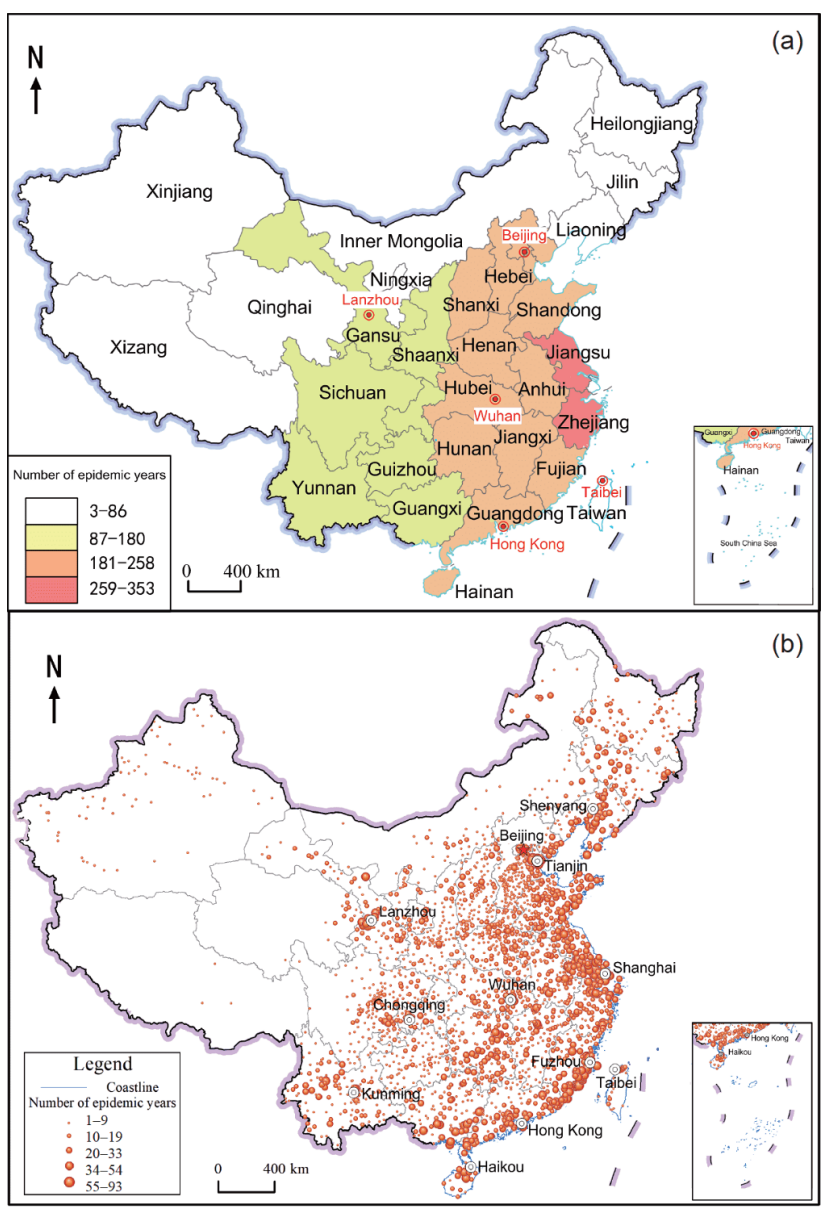

Figure 2 Spatial distribution of the number of epidemic years in China by province level ((a) 220B.C.E.-AD1949) and by county level ((b) AD1644-AD1949). The blue line indicates the modern coastline of China.

the middle and lower reaches of the Yellow River and then gradually moved southwards. During the Han and Jin dynasties, the epidemic center reached the Yangtze River and its major tributary areas. After the Tang and Song dynasties, the center expanded to the entire Yangtze River drainage and the region of Fujian and Zhejiang provinces. After the Yuan Dynasty, the center further expanded to Guangdong, Guangxi and Yunnan provinces. In the Ming Dynasty, Hainan began to experience epidemics, and in the Qing Dynasty, Taiwan, northeast China, Xinjiang, and other frontier areas were also affected.

Outbreaks of disease caused by Falciparum malaria were the oldest epidemics in China and were called "Zhang Yi". Over the past 2000 years, the northern boundary of areas in which "Zhang Yi" was prevalent has gradually moved southward. During the interval from the Warring States period to the West Han Dynasty, its northern boundary extended along the Qinling Mountain to the Huaihe River; from the Sui through to the Tang to the Five dynasties, it was along the Dabashan Mountains; and during the Ming and Qing dynasties it was along the Nanling Mountains (Gong, 1993).

\section{Discussion}

Epidemics are biological disasters resulting from the wide spread of infectious diseases caused by bacteria, viruses and parasites. The complex natural environment and living environment (Chen et al., 2019) have a major impact on the spatial and temporal distribution of epidemics. Important environmental factors such as precipitation (humidity) and dust storms can affect the frequency of epidemics via their effects on pathogens, hosts, and vectors. Study of historical epidemics in China shows that within a given year epidemics mainly occur in the relatively warm and wet summer and autumn seasons; and in terms of spatial distribution, relatively warm and humid southeastern China is more prone to epidemics. Thus the cumulative number of epidemic years exhibits the spatial pattern of a gradual decrease from southeast to northwest (Figure 2), which is highly consistent with the spatial pattern of the major attributes of China's natural environment. In terms of spatial distribution, the climate of China has not significantly changed during the past 2200 years, and therefore we calculated the correlation between average annual temperature and precipitation of counties in modern China with their respective cumulative number of epidemic years. The results showed that irrespective of historical period or region, there is a significant positive correlation.

On the millennial scale, the frequency of epidemics is significantly negatively correlated with temperature ( $r=$ $-0.334, p=0.01$ ) during the past 2200 years, which indicates that epidemics were relatively frequent in cold periods. This relationship is also evident on the centennial scale; for example, epidemics were relatively rare during warm periods, such as in the Qin-Han dynasties and the Sui-Tang dynasties, and relatively frequent during cold periods, such as in the Wei-Jin, Southern and Northern dynasties, and the MingQing dynasties (Figure 1a). Changes in the temperature anomaly record of the Northern Hemisphere and eastern China, and annual precipitation in northern China, are negatively correlated with changes in the epidemic index (Figure 1a, 1e-1g). During the past 2200 years, the importance of human activities has exceeded that of natural factors, becoming the dominant factor in determining the incidence of dust storms in northern China (Chen et al., 2020). During warm and humid periods, such as the Han and Tang dynasties and the medieval warm period, large-scale land reclamation and clearance resulted in substantial damage to the natural vegetation, leading to frequent dust storm outbreaks (Figure 1d). This contrasts with the relatively few epidemics during this period (Figure 1a).

Notably, however, climate and other environmental changes are not the dominant factors determining the frequency of epidemics. For example, the relationship between epidemics in Europe and temperature changes is not obvious (Yue and Lee, 2018). In fact, the trend of epidemic frequency 
in China during the past 2200 years is more consistent with population change (Figure 1c). Since the Five dynasties and Ten Kingdoms periods the trend of gradually increasing population in China is consistent with that of the epidemic index (Figure 1). This is because large and dense populations are more susceptible to the outbreak and spreading of epidemics. However, population size alone is not the dominant factor determining the occurrence of epidemic outbreaks. For example, the population of the Han and Tang dynasties was relatively large but the epidemic frequency was low (Figure 1a), which indicates that epidemic outbreaks were also related to social stability. During times of civil unrest and warfare (Figure 1b), such as the Wei, Jin, Southern and Northern dynasties, epidemics were more frequent. This is evidenced by the fact that on short timescales, changes in the frequency of epidemics are largely consistent with the occurrence of wars (Figure 1a). From the perspective of human history, "epidemics always follow wars" (Gong et al., 2019). In northern China, the climate was relatively warm and wet when the East Asian summer monsoon was strong. These conditions promoted the development of rainfed agriculture and resulted in periods of cultural development, social stability, and national unity, when epidemics were always relatively rare. In contrast, the climate was relatively cold and dry when the East Asian summer monsoon was weak, which resulted in frequent droughts and poor harvests, and these periods were prone to social unrest and national secession and epidemics were always relatively frequent.

Human society has always faced threats from the natural environment, amongst which epidemics caused by pathogenic microorganisms are the most important. Pathogens have evolved along with humans, with the result that epidemics have occurred throughout human history. With the development of agricultural civilization from the beginning of the Neolithic age, the human living environment has altered and generally improved. Agricultural civilization in China originated in the Yellow River drainage from which it gradually expanded outwards. With the migration of populations and shifts in civilization centers, the areas subjected to epidemics has also changed. However, in southern China, the warm and humid climatic conditions, hence the flourishing vegetation and favorable natural environment, as well as the rapidly improving living and working environment in cities and towns with large populations, were conducive to the occurrence and spread of epidemics. As a consequence, the southern region of China has been prone to major epidemics in the past and this is likely to remain in the future. With the development of civilization, the intensification of human modification of the natural environment has increased the risk of epidemics. For example, because of a misunderstanding of Chinese traditional dietary culture ("Medicines and foods are homology"), the consumption of wild animals has been and remains relatively common ( $\mathrm{Li}$ et al., 2020). This increases the risk of the spreading to humans of pathogenic microorganisms originally only found in wild animals, resulting in the outbreak and spreading of new epidemics. For example, several plagues were caused by the consumption of marmots in China. Thus a prohibition on the consumption of wild animals is likely to be an important means of promoting human development within the context of a stable natural environment, which in turn promotes human welfare and safety and hence national security. Notably, legislation was recently introduced in China banning the trade in and consumption of wild animals, and that is learning from the past can inform and shape modern social and economic policy. Further systematic study of spatiotemporal changes in past epidemics in China is likely to assist in the prevention, control, and emergency management of future epidemics.

Acknowledgements We thank the many graduate students who have participated in the collection of historical materials for the "Annals of Epidemics in China over the Past 3000 Years". We also thank Jan Bloemendal for comments and English improvement and Drs. Yajun WANG and Zimo LI for their assistance with producing the figures. This work was supported by the National Natural Science Foundation of China (Grant No. 41790421) and the National Social Science Foundation of China (Grant No. 12 \& ZD145).

\section{References}

Chen F H, Fu B J, Xia J, Wu D, Wu S H, Zhang Y L, Sun H, Liu Y, Fang X M, Qin B Q, Li X, Zhang T J, Liu B Y, Dong Z B, Hou S G, Tian L D, Xu B Q, Dong G H, Zheng J Y, Yang W, Wang X, Li Z J, Wang F, Hu Z B, Wang J, Liu J B, Chen J H, Huang W, Hou J Z, Cai Q F, Long H, Jiang M, Hu Y X, Feng X M, Mo X G, Yang X Y, Zhang D J, Wang X H, Yin Y H, Liu X C. 2019. Major advances in studies of the physical geography and living environment of China during the past 70 years and future prospects. Sci China Earth Sci, 62: 1665-1701

Gong S S. 1993. A preliminary study on variations of the distribution of Zhang-disease for the past 2000 years in China (in Chinese). Acta Geogr Sin, 48: 304-315

Gong S S. 2003. Changes of the temporal-spatial distribution of epidemic disasters in 770B.C.E.-AD1911 China (in Chinese). Acta Geogr Sin, 58: $870-878$

Gong S S. 2019. Annals of Epidemics in China over the Past 3000 Years (in Chinese). Ji'nan: Qilu Press

Gong S S, Li Z M, Liu G X, Zhang T. 2019. Temporal-spatial evolution of war-epidemic disasters in historical China (in Chinese). J Catastrophol, 34: 78-86

Chen F H, Chen S Q, Zhang X, Chen J H, Wang X, Gowan E J, Qiang M R, Dong G H, Wang Z L, Li Y C, Xu Q H, Xu Y Y, Smol J P, Liu J B. 2020. Asian dust-storm activity dominated by Chinese dynasty changes since 2000 BP. Nat Commun, 11: 992

Ge Q S, Hao Z X, Zheng J Y, Shao X M. 2013. Temperature changes over the past $2000 \mathrm{yr}$ in China and comparison with the Northern Hemisphere. Clim Past, 9: 1153-1160

Li J, Li J, Xie X R, Cai X M, Huang J, Tian X M, Zhu H. 2020. Game consumption and the 2019 novel coronavirus. Lancet Infect Dis, 20: 275-276

Yue R P H, Lee H F. 2018. Climate change and plague history in Europe. Sci China Earth Sci, 61: 163-177 